\title{
Habenular lesions and avoidance learning deficits in albino rats*
}

\author{
LYLE J. RAUSCH $\dagger$ and CHARLES J. LONG \\ Memphis State University, Memphis, Tennessee 38152
}

\begin{abstract}
Albino rats were given either habenular lesions or control operations and evaluated postsurgically during one-way active avoidance and passive avoidance training. Rats with habenular lesions, particularly those with damage in the posterior aspects of the habenular complex, were significantly impaired during acquisition of the active avoidance task. No significant differences existed between the two groups on either of two passive avoidance measures. The results are discussed in conjunction with the findings of other research, and hypotheses of either reduced fearfulness or impairment in motor inhibition mechanisms are suggested as frameworks for future research.
\end{abstract}

The functional significance of the habenular nucleus as a major waystation for reciprocal limbic midbrain connections (Herrick, 1948; Nauta, 1958, 1960) has been emphasized by recent studies finding changes in a variety of avoidance behaviors following habenular lesions. These effects on avoidance behaviors are still controversial, since different investigators have cited both deficits and increased efficiency in both active and passive avoidance learning paradigms.

Nielson and his associates (Davis, Stevenson, McIver, \& Nielson, 1966; Nielson \& McIver, 1966) found that rats with habenular lesions learned a step-down passive avoidance response faster than did controls, but were impaired in the acquisition of a conditioned active avoidance response to shock in a T-maze. On the other hand, Van Hoesen and his coworkers (Van Hoesen, MacDougall, \& Mitchell, 1969; Wilson, Mitchell, \& Van Hoesen, 1972) reported that habenular lesions impaired food-related passive avoidance, while enhancing two-way shuttle box avoidance performance. Large medial thalamic lesions, which included damage to the habenula, have been found to severely impair one-way active avoidance acquisition (Delacour, 1971; Vanderwolf, 1967, 1971), although Delacour (1971) stated that lesions limited to the habenular complex did not result in impairment in this task. Nevertheless, Tigner (1972) has reported impairment in one-way avoidance learning after lesions restricted to either the habenular nucleus or the dorsomedial thalamus.

Such discrepancies are not uncommon in the behavioral literature for several reasons, including the difficulty of replicating brain damage with the current techniques for making lesions, the changes in apparatus and shock levels from one study to another, and the variability in the responses required of the $S$ in the different behavioral situations. The present study was

\footnotetext{
*Portions of this paper were presented at the Sixth Annual Winter Conference on Brain Research, Vail, Colorado, January 1973.

†Present address: Department of Physiology, School of Medicine, University of California, Los Angeles, California 90024.
}

undertaken with the intention of minimizing these confounds by investigating the effects of habenular lesions in the same animals on two types of avoidance responding, one-way active avoidance and passive avoidance, in the same apparatus.

\section{METHOD}

\section{Subjects and Surgery}

Thirty-four Holtzman albino rats, approximately 135 days old and weighing $265-330 \mathrm{~g}$ at the time of testing, were used as Ss. All the animals were maintained on ad lib Purina Lab Chow and $15 \mathrm{~min}$ of water per day. The rats were assigned randomly to two groups, one receiving bilateral habenular lesions and the other control operations. The Ss had received prior experience in activity boxes and an odor discrimination task before avoidance training.

Surgery was performed under intraperitoneal sodium pentobarbital anesthesia (Nembutal, $50 \mathrm{mg} / \mathrm{kg}$ ) supplemented by $0.1 \mathrm{cc}(0.02 \mathrm{mg})$ atropine sulfate to suppress mucosal secretions. After a midline incision and exposure of the skull surface, a hole was drilled in the skull with a dental drill, using coordinates (AP - 2.4, DV -4.7, L 0.4) taken from the Pellegrino and Cushman (1967) brain atlas. A Grass LM4 lesion maker was used to produce thermal lesions $(1.0 \mathrm{~mA}, 15 \mathrm{sec})$ through a stainless steel electrode insulated with Insul-X except for $1.0 \mathrm{~mm}$ at the tip. Animals with control operations were subjected to similar surgical procedures, except that the electrode was lowered directly above the habenula and no current was passed through the electrode tip. After removal of the electrode, a topical antiseptic (Furacin) was applied to the skull and wound surface, the scalp was closed with wound clips, and $0.2 \mathrm{cc}$ Metrazol and 15,000 units of Bicillin were administered by intraperitoneal injections.

After the completion of data collection, the experimental animals and three controls were sacrificed under deep Nembutal anesthesia by intracardial perfusion with normal saline, followed by $10 \%$ Formalin. The brains were removed, stored in $10 \%$ Formalin for 1 day, embedded in celloidin, and sliced in 40-micron sections. The Klüver-Barrera combination stain for cell bodies and fibers was employed on every fifth section through the lesion site for evaluation of lesion extent and placement.

\footnotetext{
Apparatus

The apparatus used for both active and passive avoidance was a large wooden box with a grid floor, divided by a vertically sliding door into two compartments, one painted flat black and
} 

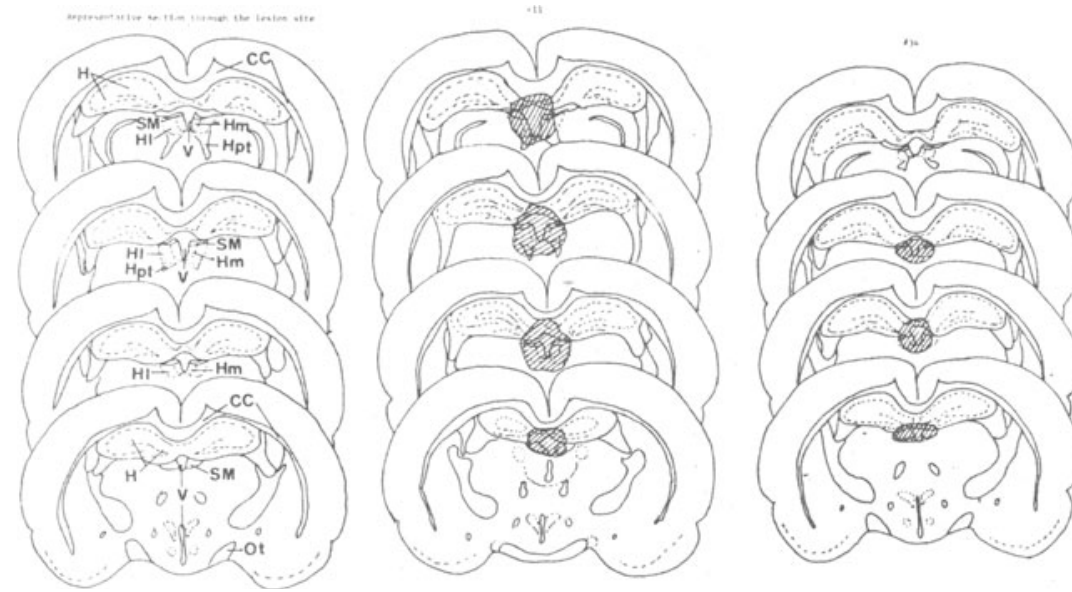

Fig. 1. Reconstructions of serial sections through the habenular area of an operated control animal and two Ss with brain damage. The first column shows a normal brain and illustrates major landmarks and habenular subdivisions; the second column shows the largest lesion found in the present study; and the third column shows an intermediate lesion with the bulk of brain damage located in the anterior habenula and the stria medullaris. Abbreviations: $\mathrm{CC}=$ corpus callosum, $\mathrm{H}=$ hippocampus, $\mathrm{Hl}=$ lateral habenula, $\mathrm{Hm}=$ medial habenula, Hpt = habenulopeduncular tract, $0 t=$ olfactory tract, $\mathrm{SM}=$ stria medullaris, $\mathrm{V}$ ventricle. the other white, and each measuring $30.5 \times 30.5 \times 15.2 \mathrm{~cm}$. The avoidance box had a small barrier, $2.5 \mathrm{~cm}$ high, across the center of the box separating the two compartments to facilitate determination of the animal's position. Clear Plexiglas removable covers were used to keep the rat from jumping out of the box. A Grason-Stadler shock source with a grid scrambler was wired to the black compartment's grid floor and programmed to deliver approximately $1.0 \mathrm{~mA}$ shock. During preliminary habituation and passive avoidance training, the sliding door was left up. All training was done under normal room illumination.

\section{Behavioral Procedures}

Active Avoidance. One-way active avoidance training began 12 days postoperatively. Avoidance training was preceded by a 15-min adaptation period in the apparatus with the sliding door left up. The animal was placed into the box straddling the middle barrier and facing the side of the apparatus. Records were kept of spontaneous crossings, defined by the location of the front feet, and total time spent in each test compartment during three 5-min timing periods. Following adaptation, the rat was placed into the black compartment facing the end wall. Five seconds later, an electric shock of about $1.0 \mathrm{~mA}$ was applied to the grid floor of the black compartment. After the rat had entered the white compartment, the sliding door was lowered and an intertrial interval of $30 \mathrm{sec}$ was allowed to elapse before the beginning of the next trial. Then the animal was placed back into the black compartment facing the end wall for the beginning of the next trial. Thirty trials were given in a single training session. Records were kept of running time and the occurrence of escape and avoidance responses with a stopwatch.

Passive Avoidance. One day after one-way active avoidance training, each rat was placed back into the white (safe) compartment of the avoidance apparatus facing the end wall. The black compartment floor was still electrified and the sliding door was left up to allow access to either compartment. The passive avoidance measure was the latency of the animal's entry into the black compartment, as measured by a stopwatch activated immediately upon the $S$ 's placement into the apparatus. The current in the black compartment was turned off only after the rat had returned to the white compartment after being shocked as a consequence of entering the black compartment. The shock received on the first entry thus provided immediate informational feedback as to the consequences associated with the black compartment.

A second latency measure was taken of the duration between the first (shocked) and the second entrance into the black compartment. If the rat failed to make a second entrance during the first 15 -min passive avoidance period, he was returned to his home cage for the rest of the day, and returned to the apparatus for $15 \mathrm{~min}$ on subsequent days until an entrance was made.

Passive avoidance ability was thus measured in two ways: (1) by recording the latency of the rat's first entrance into the electrified compartment in which he had been shocked on the previous day, and (2) by recording the latency of a second entrance into the nonelectrified black compartment after having received "self-administered" shock on the first entrance. Following passive avoidance measures, the experimental animals and three controls were sacrificed for histological verification of lesions.

\section{RESULTS}

\section{Histology}

The animals with lesions were found to have various amounts of brain damage to the habenular complex, dorsomedial thalamus, and mediodorsal hippocampus (see Fig. 1). The degree of brain damage was evaluated by three independent judges, and the experimental group was subsequently ranked according to the following criteria: (1) total habenular damage; (2) anterior habenular damage, including the stria medullaris; (3) posterior habenular damage, including the habenulo-peduncular tract; (4) dorsomedial thalamic damage; and (5) damage to the hippocampus. There was significant agreement among the judges $(p<.001$, Kendall's coefficient of concordance). The rank scores were subsequently used for rank-order comparisons of brain damage with behavioral findings.

\section{Active Avoidance}

Analysis of mean escape responses during one-way active avoidance training revealed that the experimental animals were significantly impaired $\left(\overline{\mathrm{X}}_{\mathrm{E}}=5.9, \overline{\mathrm{X}}_{\mathrm{C}}=3.8\right.$, $\mathrm{t}=3.38$, df $=32, \mathrm{p}<.01)$. The number of shocks received was significantly correlated with total habenular damage (rho $=0.505, \mathrm{t}=2.25, \mathrm{df}=15, \mathrm{p}<.05)$ and damage to the posterior habenula (rho $=0.593, \mathrm{t}=2.85$, $\mathrm{df}=15, \mathrm{p}<.02$ ). Correlations with anterior habenular, hippocampal, and dorsomedial thalamic damage were not significant. No significant differences existed between the two groups on either mean escape latencies $(6.10 \mathrm{sec} ; 6.06 \mathrm{sec})$ or mean avoidance latencies $(1.72 \mathrm{sec} ; 1.75 \mathrm{sec})$. The data are summarized in Table 1.

\section{Passive Avoidance}

Two passive avoidance latency scores were analyzed: (1) latency of initial entrance into the black 
compartment after placement into the white compartment $24 \mathrm{~h}$ after active avoidance training, and (2) latency of return to the black compartment after receipt of shock on the first entrance into the black compartment. The scores are summarized in Table 1.

There were no significant differences between the first latency scores of the two groups $\left(\operatorname{median}_{\mathrm{E}}=115 \mathrm{sec}\right.$, median $_{\mathbf{C}}=90 \mathrm{sec}$, Mann-Whitney U test, n.s.). All the animals returned to the black compartment relatively quickly. First latency scores for the experimental groups were correlated with damage to the anterior habenula (rho $=-0.523, \mathrm{~N}=17, \mathrm{p}<.05$ ), but not with any other histological criterion. Due to an equipment failure during which the shock was turned off momentarily in the black compartment, only 13 experimental animals and 16 controls were tested on the second latency measure. No significant differences were obtained between the two groups $\left(\operatorname{median}_{E}=40.9 \mathrm{~min}\right.$, median $_{C}=34.0 \mathrm{~min}$, Mann-Whitney U test, n.s.). Second latency scores were not significantly correlated with any histological criterion, nor were individual scores on the two latency measures correlated with each other. However, second latencies were considerably longer than first latencies, comprising minutes rather than seconds. Planned comparisons of spontaneous crossings in the box before and after shock in the black compartment were not statistically analyzed because the information-providing shock on the first passive avoidance entry resulted in total avoidance of the shocked compartment for periods ranging from 10.6 to $66.1 \mathrm{~min}$.

\section{DISCUSSION}

Heimer (1972) has recently described a fiber system leading from the pyriform cortex and amygdala to the mediodorsal thalamus, which projects through the stria medullaris and habenula. While this system is of unknown functional significance, it should be kept in mind that the results of this and other studies of the effects of habenular lesions may be due to an interruption of this pathway rather than to the destruction of habenular neurons.

The present findings that rats with habenular lesions accept more shocks than controls while learning one-way active avoidance corroborate earlier research by Tigner (1972). This behavioral impairment can be variously ascribed to a general deficit in learning ability, reduced fearfulness, motor impairment, or some combination of these factors. A generalized learning deficit is not supported by the present data, however, since rats with habenular lesions performed as well as controls in the passive avoidance tests. There are other indications that these rats are not simply poor learners, since they can learn operant discriminations with ease (Eaton, Rausch, Rausch, \& Long, 1972; Rausch, Rausch, \& Long, 1971) and in some paradigms learn avoidance responses at a rate faster than do controls (Davis et al, 1966; Van Hoesen et al, 1969). The following discussion proposes that the effects of habenular lesions are best explained by positing reduced fearfulness, possibly accompanied by some impairment in the initiation of new behaviors.

What rationale exists for assuming that habenular lesions reduce fearfulness? Lowered emotional reactivity would be expected to reduce the animal's response to shock and hence to result in less motivation to learn the one-way active avoidance task in the present study. Previous studies which support this contention include the findings that rats with habenular lesions extinguish a conditioned emotional response (CER) faster than controls (Brady \& Nauta, 1955), ambulate more in an open field (Nielson \& McIver, 1966), and are less likely to attempt to escape from a step-down platform (Davis et al, 1966). Reduced fearfulness can also be invoked to account for the apparently contradictory findings that rats with habenular lesions are better at shuttlebox avoidance (Van Hoesen et al, 1969) but worse at avoidance learning in a T-maze (Nielson \& McIver, 1966), since in the former task fear of returning to the compartment in which shock has been experienced is presumed to retard the learning rate of controls. Moreover, Reinert (1964) has described the effects of habenular stimulation in cats as producing a behavioral pattern which resembles fear rather than rage or aggression. There is also physiological evidence linking the habenula and the adrenal gland (Farrell \& Taylor, 1962; Faure, LeNouene, Vincent, \& Bensch, 1965a, b), which suggests the possibility of hormonal mechanisms underlying the hypothesized state of reduced fearfulness.

Alternately, the deficits in active avoidance behavior might be due to an impairment in motor systems. In this context, it is useful to consider what Vanderwolf (1967, 1971) terms "trigger" mechanisms for initiating voluntary movement. It is possible that habenular lesions

Table 1

Summary of Avoidance Data

\begin{tabular}{|c|c|c|c|c|c|c|c|c|c|}
\hline \multirow[b]{3}{*}{ Group } & \multicolumn{3}{|c|}{ Active Avoidance } & \multicolumn{6}{|c|}{ Passive Avoidance } \\
\hline & \multicolumn{3}{|c|}{ Escapes } & \multicolumn{3}{|c|}{ Latency I (Sec) } & \multicolumn{3}{|c|}{ Latency II (Min) } \\
\hline & $\mathbf{N}$ & Mean & Range & $\mathbf{N}$ & Median & Range & $\mathrm{N}$ & Median & Range \\
\hline Experimental & 17 & $5.9 *$ & $4-12$ & 17 & 115 & $2-794$ & 13 & 40.9 & $10.6-66.1$ \\
\hline Control & 17 & 3.8 & $2-5$ & 17 & 90 & $7-198$ & 16 & 34.0 & $16.6-60.0$ \\
\hline
\end{tabular}

${ }^{*} p<.01$ 
modify the trigger mechanisms by which one response system or motor set gives way to another. Such an impairment would make an ongoing response less amenable to being "switched," and thus less likely to yield precedence to another response. Since the considerable flexibility of the central nervous system is a function of many variables, including motivational levels, response complexity, stimulus saliency, past experience, redundancy of connections, etc., a switch in motor responses would eventually occur, but more input than normal might be required to effect a switch from one response to another. Thus the shock intensity employed in the present study may have been less effective in triggering the switching mechanisms which facilitate the correct response of running into the safe chamber during one-way avoidance training. If this were the case, however, increased latencies of escape during active avoidance would be expected, but although the animals with lesions made fewer avoidance responses than controls, their response latencies on both escape and avoidance trials were in the same range as controls.

Nevertheless, it would be premature to dismiss the possibility of some kind of inhibitory deficit interacting with reduced fearfulness in these and other findings. For example, the impairments in shock avoidance observed by Nielson and McIver (1966) in the T-maze situation, which had a position-reversal component, were considered by the authors to be related to inhibitory deficits. Their rats would run to an "incorrect" bar in the maze and then turn around and run directly to the "correct" bar and press it. The Davis-Van Hoesen controversy is also susceptible to solution by relating it to motor inhibition. Davis et al (1966), using a step-down task, found that rats with lesions were better at passively avoiding shock than were controls (i.e., they stayed on the platform longer). However, Van Hoesen et al (1969) and Wilson et al (1972) reported impairment in passive avoidance of shock paired with food consumption (i.e., rats with habenular lesions continued to eat after being shocked while eating). The inhibition hypothesis can account for both findings on the basis of a tendency to continue ongoing behavior, although one must specify which of the two incompatible responses (stepping down or staying on the platform; eating or not eating) is to be considered as prepotent or ongoing in each situation. Davis et al observed that the initial latency of stepping down prior to being shocked was greater for animals with habenular lesions than for controls, suggesting that the ongoing response for controls of stepping off the platform had, itself, been altered by the lesions. Of course, reduced emotional reactivity could be invoked to suggest that the animals with lesions in the step-down task were less motivated to attempt to escape from the platform, and hence performed "better," while the animals shocked while eating were less motivated by the fear of shock than by the positive motivation of food and hence performed "worse" in this type of passive avoidance. Further clarification could be obtained by establishing the appropriate control conditions during future studies.

The passive avoidance data in the present study did not reveal any differences between the two groups. While these findings indicate that rats with habenular lesions are capable of acquiring adaptive behavior in aversive situations, they are inconsistent with previous research which has found either impairment (Van Hoesen et al, 1969; Wilson et al, 1972) or enhancement (Davis et al, 1966) of passive avoidance behavior in animals with habenular lesions. However, the present negative results may be a function of differential active avoidance experience, since the independent variable for producing passive avoidance was the shock received during active avoidance training, which was different for the two groups. Additional research would be necessary to assess adequately the contribution of decreased fearfulness to the present results. This could be accomplished by evaluating passive avoidance after each group had received the same number of unavoidable shocks, or by making pre- and postoperative determinations of shock thresholds in a conditioned suppression paradigm.

Further studies will be necessary to clarify the issues, but the present results, in conjunction with previous research, suggest that lesions in the habenular area result in: (1) reduced emotional reactivity, (2) a tendency to continue ongoing behavior, or (3) some combination of these factors. It appears more fruitful at the present time to pursue hypotheses designed to reconcile apparently divergent findings than to assume that some experimental findings are reliable while others are not.

\section{REFERENCES}

Brady, J. V., \& Nauta, W. J. H. Subcortical mechanisms in emotional behavior: The duration of affective changes following septal and habenular lesions in the albino rat. Journal of Comparative \& Physiological Psychology, 1955, 48, 412-420.

Davis, K. B., Stevenson, M., McIver, A. H., \& Nielson, H. C. The effect of a lesion of the habenula on passive avoidance learning. Psychonomic Science, 1966, 5, 5-6.

Delacour, J. Effects of medial thalamic lesions in the rat. A review and an interpretation. Neuropsychologia, 1971, 9, 157-174.

Eaton, N. R., Rausch, L. J., Rausch, H. R., \& Long, C. J. Olfactory discrimination and reversal learning in rats with limbic lesions. Paper presented at the Southeastern Psychological Association Convention, Atlanta, April 1972.

Farrell, G., \& Taylor, A. N. Neuroendocrine aspects of blood volume regulation. Annual Review of Physiology, 1962, 24, 471-490.

Faure, J., LeNouène, J., Vincent, J. D., \& Bensch, C. Influence des destructions de l'habenula sur l'elimination urinaire du sodium. Journal de Physiologie, 1965a, 57,610.

Faure, J., LeNouène, J., Vincent, J. D., \& Bensch, C. Réactions bio-électriques de l'habenula et du tronc cérébral a l'hypovolémie expérimentale. Revue Neurologique, 1965b, 112, 258-266.

Heimer, L. The olfactory connections of the diencephalon in the rat. Brain, Behavior \& Evolution, 1972, 6, 484-523.

Herrick, C. J. The brain of the tiger salamander. Chicago: University of Chicago Press, 1948. 
McCleary, R. A. Response-modulating functions of the limbic system: Initiation and suppression. In $\mathbf{E}$. Stellar and J. M. Sprague (Eds.), Progress in physiological psychology. Vol. 1. New York: Academic Press, 1966.

Nauta, W. J. H. Hippocampal projections and related neural pathways to the midbrain in the cat. Brain, 1958, 81, 319-340.

Nauta, W. J. H. Some neural pathways related to the limbic system. In E. R. Ramey and D. S. Doherty (Eds.), Electrical studies of the unanesthetized brain. New York: Hoeber, 1960.

Nielson, H. C., \& McIver, A. H. Cold stress and habenular lesion effects on rat behaviors. Journal of Applied Physiology, 1966, 21, 655-660.

Pellegrino, L. J. \& Cushman, A. J. A stereotaxic atlas of the rat brain. New York: Appleton-Century-Crofts, 1967.

Rausch, L. J., Rausch, R., \& Long, C. J. Effect of habenular lesions on food od or preference and food consumption in rats. Paper presented at the First Annual Meeting of the Society for Neuroscience, Washington, D.C., October 1971.

Reinert, H. Defence reaction from the habenular nuclei, stria medullaris and fasciculus retroflexus. Journal of Physiology, $1964,170,28-29 P$.
Tigner, J. C. Impairment of one-way active avoidance in rats with habenular or dorsomedial thalamic lesions. Psychonomic Science, 1972, 27, 7-8.

Vanderwolf, C. H. Effects of experimental diencephalic damage on food hoarding and shock avoidance behavior in the rat. Physiology \& Behavior, 1967, 2, 399-402.

Vanderwolf, C. H. Limbic-diencephalic mechanisms of voluntary movement. Psychological Review, 1971, 78, 83-113.

Van Hoesen, G. W., MacDougall, J. M., \& Mitchell, J. C Anatomical specificity of septal projections in active and passive avoidance behavior in rats. Journal of Comparative \& Physiological Psychology, 1969, 68, 80-89.

Wilson, J. R., Mitchell, J. C., \& Van Hoesen, G. W. Epithalamic and ventral tegmental contributions to avoidance behavior in rats. Journal of Comparative \& Physiological Psychology, $1972,78,442-449$.

(Received for publication August 20, 1973; revision received May 9,1974 .) 